\title{
Poliquetos asociados con Austromegabalanus psittacus (Molina, 1782) (Crustacea: Cirripedia) en Península Gualpén, Chile central: Biodiversidad y efecto del tamaño del sustrato biológico.
}

\author{
Polychaetes associated with Austromegabalanus psittacus (Molina, 1782) (Crustacea: Cirripedia) on \\ the Peninsula Gualpén, central Chile: Biodiversity and the effect of biological substrate size. \\ Cristián E. Hernández ${ }^{1,2}$, Gabriela Muñoz ${ }^{1}$ y Nicolás Rozbaczylo ${ }^{2}$ \\ ${ }^{1}$ Facultad de Ciencias, Universidad Católica de la Ssma. Concepción. Casilla 297, Concepción \\ ${ }^{2}$ Departamento de Ecología, Facultad de Ciencias Biológicas, Pontificia Universidad Católica de Chile, Casilla 114-D, Santiago \\ chernand@genes.bio.puc.cl
}

\begin{abstract}
Resumen.- Se estudió la asociación de poliquetos con el cirripedio Austromegabalanus psittacus en la Península Gualpén (VIII Región, Chile). Se informa y discute la importancia de esta asociación para los ensambles de poliquetos submareales, y se indaga sobre la importancia que tiene el tamaño corporal del cirripedio en la abundancia y biodiversidad de poliquetos. Todos los cirripedios examinados tenían poliquetos, obteniéndose 793 individuos pertenecientes a 19 especies de 12 familias. La mayoría de los poliquetos se encontraron en la parte calcárea de los cirripedios. Los más abundantes y frecuentes fueron Syllidae (Typosyllis sp., Autolytus sp.), Nereididae (Nereis callaona), Spionidae (Polydora sp.), Chrysopetalidae (Paleanotus chrysolepis) y Cirratulidae (Dodecaceria opulens). Se observó que la biodiversidad de poliquetos y la abundancia de Typosyllis sp. aumentan significativamente con el tamaño del cirripedio. La relación entre el tamaño de $A$. psittacus y la riqueza de poliquetos está determinada por la ecuación potencial $(\mathrm{Y}=$ $\mathrm{cX}^{\mathrm{z}}$ ) que empíricamente describe la conocida relación especies-área en el modelo de equilibrio de MacArthur \& Wilson (1963), por lo que los cirripedios se comportarían como islas para los poliquetos asociados. Se discute la importancia de los poliquetos registrados en relación con asociaciones semejantes en otras costas del mundo.

Palabras claves: Asociación faunistica, anélidos, poliquetofauna, submareal somero, biogeografía de islas.
\end{abstract}

\begin{abstract}
We studied the association of polychaetes with the cirriped, Austromegabalanus psittacus on the Peninsula of Gualpén (VIII Region, Chile). Here we report and discuss the importance of this association for assemblages of subtidal polychaetes, we also investigated about the importance of cirriped body size on the abundance and biodiversity of polychaetes. All of the cirripeds examined contained polychaetes. We obtained 793 individuals belonging to 19 species of 12 families. The majority of the polychaetes were found in the calcareous part of the cirripeds. The most abundant and prevalent were: Sylidae (Typosyllis sp., Autolytus sp.), Nereididae (Nereis callaona), Spinonidae (Polydora sp.), Chyrospetalidae (Paleanotus chrysolepis) and Cirratulidae (Dodecaceria opulens). We observed that the biodiversity of polychaetes and the abundances of Typosyllis sp. significantly increased with the size of the cirriped host. The relationship between the size of A. psittacus and the richness of polychaete species is explained by the potential equation $\left(\mathrm{Y}=\mathrm{c} \mathrm{X}^{\mathrm{z}}\right)$, which empirically describes the known ratio species-area in the equilibrium model of MacArthur \& Wilson (1963), where the cirripeds behave like islands for the associated polychaetes. We discuss the importance of the recorded polychaetes in relation to similar associations on other coasts of the world.

Key words: Faunistic association, annelids, polychaete fauna, shallow subtidal, island biogeography.
\end{abstract}

\section{Introducción}

Los poliquetos constituyen en general uno de los taxa más importante en las comunidades marinas de fondos blandos (Oyarzún et al. 1987, Cañete et al. 1999) y duros (Alós 1990) en cuanto a número de especies e individuos. También se han señalado como uno de los grupos más abundantes y diversos formando asociaciones con otros organismos (Zamorano \& Moreno 1975, Paredes \& Tarazona 1980, Villouta \&
Santelices 1984, López \& Stotz 1997). Por este motivo, el conocimiento de la poliquetofauna en las diferentes biocenosis del litoral es considerado importante para caracterizar los distintos hábitat bentónicos (Alós et al. 1982) e incluso para programas de vigilancia ambiental (Cañete et al. 2000), al constituir especies sensitivas o indicadoras de contaminación (Carrasco \& Gallardo 1989).

La asociación de poliquetos con otros organismos presenta gran interés, ya que según algunos autores 
estas asociaciones explicarían las variaciones batimétricas de la diversidad en el submareal somero (Ojeda \& Dearborn 1989). Por lo que la presencia y las características de estos sustratos biológicos tendrían gran importancia para la biodiversidad de poliquetos. En este sentido, si consideramos que los sustratos biológicos son hospedadores que se comportan como islas para las especies que alojan (Kuris et al. 1980), se espera que el número de especies de poliquetos que soporta el sustrato biológico aumente con el tamaño corporal de este, tal como se esperaría para la relación especie-área en biogeografía de islas (MacArthur \& Wilson 1963, Gotelli 1995, Brown \& Lomolino 1998, Jaksic 2001). En particular, para los organismos de cuerpo blando, como los poliquetos (Barnes 1987), los sustratos biológicos podrían constituir un refugio en constante crecimiento en cual se realicen aquellos eventos de la historia de vida (ej. reproducción) que en vida libre podrían tener menor probabilidad de ocurrencia, por ejemplo por depredación. Al respecto, se ha observado que poliquetos de vida libre de los géneros Pseudonereis Kinberg, 1866 y Platynereis Kinberg, 1866 cuando se encuentran reproductivamente maduros, al parecer, utilizan los intersticios de las agregaciones de tubos del poliqueto Pharagmatopoma moerchi Mörch, 1863 en la zona norte de Chile (Cañete, com. pers.).

Existen trabajos sobre poliquetos asociados a esponjas (Alós et al. 1982, Cinar \& Ergen 1998), tunicados (Zamorano \& Moreno 1975), corales (Tsuchiya et al. 1986), algas (Alós 1983, Vásquez \& Santelices 1984, Villouta \& Santelices 1984, Ojeda \& Santelices 1984, López \& Stotz 1997), bivalvos (Paredes \& Tarazona 1980, Tsuchiya \& Hirano 1985, Tsuchiya \& Nishihira 1985, 1986, Feller et al. 1992) y a agregaciones de tubos de otros poliquetos (Zamorano $2000)^{1}$. De ellos 6 se han realizado en Chile, destacándose la importancia de 5 sustratos biológicos en la biodiversidad de poliquetos para la costa de Chile: el tunicado Pyura chilensis Molina, 1782 (Zamorano \& Moreno 1975), las algas pardas Lessonia nigrescens Bory, 1826 (Vásquez \& Santelices 1984, Villouta \& Santelices 1984) y Macrocystis pyrifera (Linnaeus) (Ojeda \& Santelices 1984), el alga Corallina officinalis (Decaisne) (López \& Stotz 1997) y la agregación de tubos del poliqueto Pharagmatopoma moerchi (Zamorano 2000) ${ }^{1}$.

No existen hasta el momento trabajos sobre la infracomunidad de poliquetos asociados a crustáceos

1 Zamorano JH. 2000. Fauna asociada a las agregaciones del poliqueto tubícola Phragmatopoma virgini Kinberg, en el intermareal rocoso de Mehuin. XX Congreso de Ciencias del Mar, Concepción, Chile, p. 217, Resumen. cirripedios, un importante componente de las comunidades de macroinvertebrados en las zonas litorales del mundo (Barnes \& Hughes 1990; Little \& Kitching 1996) y un potencial hábitat para los poliquetos horadadores o perforadores de placas de carbonato de calcio, como por ejemplo, para representantes de la familia Spionidae (ej. Polydora sp.) (Rozbaczylo 1985). Por lo tanto, su estudio podría aportar al entendimiento de patrones de diversidad y abundancia de poliquetos en diferentes comunidades litorales del mundo.

En este trabajo se identifica y cuantifica la poliquetofauna asociada al cirrepedio no pedunculado de mayor tamaño en el mundo (Darwin, 1854) Austromegabalanus psittacus (Molina, 1782), lo que se traduce en una oferta de sustrato para los poliquetos no estudiada hasta ahora. Destacando, además, que $A$. psittacus es una especie comercial del submareal somero de Chile (SERNAP 1990-1999, Bustamante \& Castilla 1987) y quizás la única en el mundo. Con el objeto de informar y discutir la importancia de $A$. psittacus para los ensambles de poliquetos submareales, se indaga sobre la abundancia y la biodiversidad de poliquetos, y el efecto que tiene en ellos el tamaño corporal del cirripedio (tamaño del sustrato biológico).

\section{Materiales y Métodos}

En la costa norte de la Península de Gualpén (3644’S, 73¹1’ W), VIII Región (Chile), durante enero de 1998 se extrajo 30 individuos de $A$. psittacus, obtenidos en un muestreo al azar mediante buceo autónomo (SCUBA) en el submareal somero (4 a 10 metros de profundidad). Como indicador de tamaño corporal se usó el área opercular de cada cirripedio, medida de acuerdo a la metodología de Hernández (1999), ya que este indicador es una medida denso-independiente del tamaño corporal y la edad de A. psittacus (López 1994).

Para extraer los poliquetos, se consideró la estructura corporal de los cirripedios, separando cada individuo en tres zonas de análisis: interior, concha y placas operculares. La recolección de los poliquetos del interior consistió en abrir el animal y revisar bajo lupa estereoscópica Wild M5 el agua contenida y los tejidos blandos. Las conchas de cada individuo se revisaron in situ extrayendo los poliquetos de mayor tamaño, luego las conchas y las placas operculares fueron puestas por separado en recipientes plásticos con formalina al $5 \%$, para desprender y preservar los poliquetos de menor tamaño. Posteriormente, se separó y recogió los poliquetos bajo lupa estereoscópica, lavando y filtrando el contenido de los recipientes con un tamiz de $300 \mu \mathrm{m}$ de apertura bajo un chorro de agua a presión. Los poliquetos extraídos de las zonas de análisis fueron guardados en formalina al $10 \%$. Para determinar la 
composición de poliquetos total y por cirripedio, las familias y géneros se establecieron según Fauchald (1977). Se dibujaron los taxa más abundantes por medio de una cámara lúcida instalada sobre una lupa estereoscópica Wild M5.

Para cuantificar la poliquetofauna asociada a $A$. psittacus, se determinó la abundancia individual (número de poliquetos de cada taxón por cirripedio), la frecuencia de aparición porcentual (\% de cirripedios que presentó cada taxa de poliqueto) y la abundancia media (número de poliquetos de cada taxón dividido por el número de cirripedios totales de la muestra). Para determinar la importancia de A. psittacus para los poliquetos asociados, se realizó un análisis de biodiversidad alfa utilizando el software Biodiversity Profesional Versión 2 (McAleece 1997). En este programa se determinó para la muestra total y por cirripedio: 1.- la riqueza de especies $\mathrm{R}$ (número de especies); 2.- la diversidad H' y uniformidad J' de Shannon-Weaver (Shannon \& Weaver 1949); 3.- la diversidad D de Simpson (Simpson 1949); y 4.- la diversidad $\mathrm{N}_{1}$ de Hill (Hill 1973). Los índices de Shannon-Wiener y Simpson se escogieron por ser unos de los más populares en la literatura (Jaksic 2001) y por lo tanto permiten establecer comparaciones con otros trabajos de características similares. Sin embargo, estos índices le dan demasiada importancia a las especies abundantes, despreciando la presencia de las especies raras. Por esta razón, se usó el índice de Hill, debido a que entrega un valor recíproco medio proporcional a la abundancia y clasifica de acuerdo al peso que tienen las especies raras (Hill 1973).

El efecto del tamaño del cirripedio sobre la biodiversidad de poliquetos, se estimó graficando el número de familias, número de especies, e índice de diversidad de Hill $\left(\mathrm{N}_{1}\right)$, con respecto al área opercular del cirripedio. La importancia del tamaño corporal sobre la abundancia de poliquetos se determinó graficando la abundancia total y por taxón (sólo para los taxa con mayor abundancia y frecuencia de aparición), con respecto al área opercular del cirripedio. Para describir la tendencia en cada relación, se buscó la curva con mayor coeficiente de determinación $\left(\mathrm{R}^{2}\right)$, calculando la significancía de la pendiente a través de un análisis de la varianza (Zar, 1996).

\section{Resultados}

Los cirripedios analizados presentaron tamaños que fluctuaron entre 0,3 y 20,9 $\mathrm{cm}^{2}$ de área opercular (Promedio $=7,17 \pm 4,49$ DE), y entre 0,5 y 171,7 g de peso corporal en partes blandas (Promedio $=63,08 \pm$ 40,08 DE). Todos los cirripedios presentaron poliquetos asociados, obteniéndose 793 individuos pertenecientes a 19 especies y 12 familias (Tabla 1). En la Tabla 1 se indican las características biológicas del gremio de alimentación para las especies de acuerdo a la clasificación de Fauchald \& Jumars (1979); se observan variados tipos de alimentación, de estructura utilizada para ello y de movilidad, destacando por su abundancia y riqueza los poliquetos carnívoros, con mandíbula y móviles. Del total de poliquetos, 8 individuos (1 \%) pertenecientes a dos especies (2 individuos de Typosyllis sp. y 6 de Nereis callaona (Grube, 1857)) se encontraron en la parte interior de las placas del cirripedio y el resto (99 \%) en la parte externa calcárea (placas operculares y concha). Sin embargo, estos son dos de los más abundantes en la parte calcárea, por lo que todos los análisis se realizaron para el conjunto de poliquetos por cirripedio.

Los taxa más abundantes fueron representantes de las familias Syllidae que constituyeron el 59 \% de la abundancia total (Autolytus sp. y Typosyllis sp.), Spionidae (Polydora sp.) y Nereididae ambas con $11 \%$ (Nereis callaona), Chrysopetalidae con 7 \% (Paleanotus chrysolepis Schmarda, 1861) y Cirratulidae con $4 \%$ (Dodecaceria opulens Gravier, 1908) (Tabla 1, Fig. 1). El análisis de biodiversidad alfa (Tabla 2) permite señalar que $A$. psittacus sustenta una alta abundancia y diversidad de poliquetos asociados, lo cual se refleja en los valores de los índices de Shannon (H'), Simpson (D) y Hill $\left(\mathrm{N}_{1}\right)$, siendo este último el que mejor representa la riqueza de especies $(\mathrm{R})$ observada (19 taxa).

La biodiversidad de los poliquetos varía en forma de una curva potencial con respecto al tamaño del cirripedio (Fig. 2), dado que esta relación ( $\mathrm{Y}=\mathrm{cX}^{\mathrm{z}}$, donde c es el intercepto y z la pendiente) en todos los casos mostró el máximo valor de coeficiente de determinación $\left(\mathrm{R}^{2}\right)$ y pendientes significativamente distintas de cero ( $\mathrm{p}<0,0001)$. Al respecto, se observa que el tamaño del sustrato biológico explica el $78 \%$ de la variabilidad en el número de familias, el $76 \%$ en el número de especies, y el 51 \% en el índice de diversidad de Hill $\left(\mathrm{N}_{1}\right)$. Cuyas pendientes ( $\mathrm{z}$ entre 0,49 y 0,52 ) indican en todos los casos, que existe un aumento rápido de la diversidad en tamaños pequeños del sustrato y una tendencia a la saturación en tamaños mayores (Fig. 2). Del mismo modo, el área opercular del cirripedio se relaciona en forma potencial con la abundancia total de poliquetos $\left(\mathrm{R}^{2}=0,67\right)$, aunque no se observa una tendencia a la saturación de individuos, los cuales aumentan casi directamente proporcional al tamaño del cirripedio (pendiente $\mathrm{z}=0,91$ ). Por otra parte, la abundancia individual de poliquetos mostró una relación significativa con el área sólo en Typosyllis sp. $(\mathrm{p}=0,01)$ (Fig. 3), con un $20 \%$ de la variabilidad de la abundancia explicada por el tamaño del cirripedio, en cambio en los otros taxa no se encontraron relaciones significativas $(\mathrm{p}>0,05)$. 
Tabla 1

Número de poliquetos (N), abundancia media (A) y frecuencia porcentual (F \%) de las 19 taxa de poliquetos asociados a $A$. psittacus. Además, para cada familia se indica el número de poliquetos ( $\mathrm{Nf}$ ), la riqueza de especies (R), y las características biológicas de las especies del gremio de alimentación de acuerdo a la clasificación de Fauchald \& Jumars (1979). Donde Al es el tipo de alimentación $(C=$ Carnívoros, $S=$ Se alimentan de depósitos superficiales, $B=$ Se alimentan de depósitos subsuperficiales, $\mathbf{O}=$ Omnívoros, y $\mathbf{F}=$ Filtrador), EA estructura utilizada para alimentación $(\mathbf{M}=\mathbf{M a n d i ́ b u l a s , ~} \mathbf{F E}=$ Faringe eversible, y $\mathbf{T}$ = Tentáculos) y M movilidad ( $M$ = móvil, $\mathrm{DM}=$ Discretamente móvil, y $\mathrm{S}=$ Sésil).

Number of polychaetes (N), average abundance (A), and percent frequency (F \%) of the 19 taxa of polychaetes associated with $A$. psittacus. Also, for each family we indicate the number of polychaetes (Nf), species richness (R), and the biological characteristics of the species of feeding guilds according to the classification by Fauchald \& Jumars (1979). Where Al is the type of feeding ( $\mathrm{C}=$ Carnivores, $\mathrm{S}=$ Superficial deposit feeders, $\mathrm{B}=$ Subsuperficial deposit feeders, $\mathrm{O}=$ Omnivores, and $\mathrm{F}=$ filter feeders), EA is the structure used for feeding $(\mathrm{M}=$ Mandibles, $\mathrm{FE}=$ Eversible Pharynges, and $\mathrm{T}=$ Tentacles $)$, and $\mathbf{M}$ mobility $(\mathbf{M}=\mathrm{mobile}, \mathrm{DM}=$ discretely mobile, and S = Sessile).

\begin{tabular}{|c|c|c|c|c|c|c|c|c|}
\hline \multirow[b]{2}{*}{ TAXA } & \multirow[b]{2}{*}{$\mathbf{N}$} & \multirow[b]{2}{*}{$\mathbf{A}$} & \multirow[b]{2}{*}{ F \% } & \multirow[b]{2}{*}{ Nf } & \multirow[b]{2}{*}{$\mathbf{R}$} & \multicolumn{3}{|c|}{ Características biológicas } \\
\hline & & & & & & Al & EA & $\mathbf{M}$ \\
\hline SYLLID AE & & & & 469 & 4 & & & \\
\hline Typosyllis sp. & 386 & 12.87 & 93.3 & & & & & \\
\hline Autolytus sp. & 66 & 2.20 & 63.3 & & & $\mathrm{C}$ & M & M \\
\hline Amblyosyllis sp. & 2 & 0.07 & 6.7 & & & & & \\
\hline Trypanosyllis sp. & 15 & 0.50 & 33.3 & & & & & \\
\hline $\begin{array}{l}\text { CHR YSO PET AL ID AE } \\
\text { Paleanotus chrysolepis Schmarda, } 1861\end{array}$ & 57 & 1.90 & 90.0 & 57 & 1 & $\mathrm{C}$ & FE & M \\
\hline $\begin{array}{l}\text { CIRRATULIDAE } \\
\text { Dodecaceria opulens Gravier, } 1908\end{array}$ & 31 & 1.03 & 56.7 & 31 & 1 & S & $\mathrm{T}$ & S \\
\hline $\begin{array}{l}\text { NEREIDIDAE } \\
\text { Nereis callaona (Grube, 1857) } \\
\text { Perinereis nuntia vallata (Grube, 1857) }\end{array}$ & $\begin{array}{c}86 \\
1\end{array}$ & $\begin{array}{l}2.87 \\
0.03\end{array}$ & $\begin{array}{c}93.3 \\
3.3\end{array}$ & 87 & 2 & $\mathrm{O}$ & M & M \\
\hline $\begin{array}{l}\text { SABELLIDAE } \\
\text { Sabellidae indet. }\end{array}$ & 5 & 0.17 & 16.7 & 5 & 1 & $\mathrm{~F}$ & $\mathrm{~T}$ & $S$ \\
\hline $\begin{array}{l}\text { EUNIC ID AE } \\
\text { Marphysa aenea (Blanchard, 1849) } \\
\text { Lysidice sp. }\end{array}$ & $\begin{array}{l}2 \\
1\end{array}$ & $\begin{array}{l}0.07 \\
0.03\end{array}$ & $\begin{array}{l}6.7 \\
3.3\end{array}$ & 3 & 2 & $\mathrm{O}$ & M & M \\
\hline $\begin{array}{l}\text { PHY LOD OC IDAE } \\
\text { Eulalia sp. }\end{array}$ & 28 & 0.93 & 40.0 & 28 & 1 & $\mathrm{C}$ & $\mathrm{FE}$ & M \\
\hline $\begin{array}{l}\text { SPIONID AE } \\
\text { Polydora sp. } \\
\text { Boccardia tricuspa (Hartman, 1939) } \\
\text { Spiónidos indet. }\end{array}$ & $\begin{array}{c}88 \\
1 \\
2\end{array}$ & $\begin{array}{l}2.93 \\
0.03 \\
0.07\end{array}$ & $\begin{array}{c}66.7 \\
3.3 \\
6.7\end{array}$ & 91 & 3 & F-S & $\mathrm{T}$ & $\mathrm{DM}$ \\
\hline $\begin{array}{l}\text { POLYNOIDAE } \\
\text { Polynoidae indet. }\end{array}$ & 11 & 0.37 & 33.3 & 11 & 1 & $\mathrm{C}$ & M & M-D M \\
\hline $\begin{array}{l}\text { TEREBELID AE } \\
\text { Streblosoma sp. }\end{array}$ & 9 & 0.30 & 26.7 & 9 & 1 & S & $\mathrm{T}$ & $S$ \\
\hline $\begin{array}{l}\text { LUMBR IN ER ID AE } \\
\text { Lumbrineris sp. }\end{array}$ & 1 & 0.03 & 3.3 & 1 & 1 & $\mathrm{O}$ & M & M \\
\hline $\begin{array}{l}\text { SCALIBRE G MATIDAE } \\
\text { Hyboscolex sp. }\end{array}$ & 1 & 0.03 & 3.3 & 1 & 1 & B & FE & M \\
\hline Suma & 793 & & & 793 & 19 & & & \\
\hline
\end{tabular}




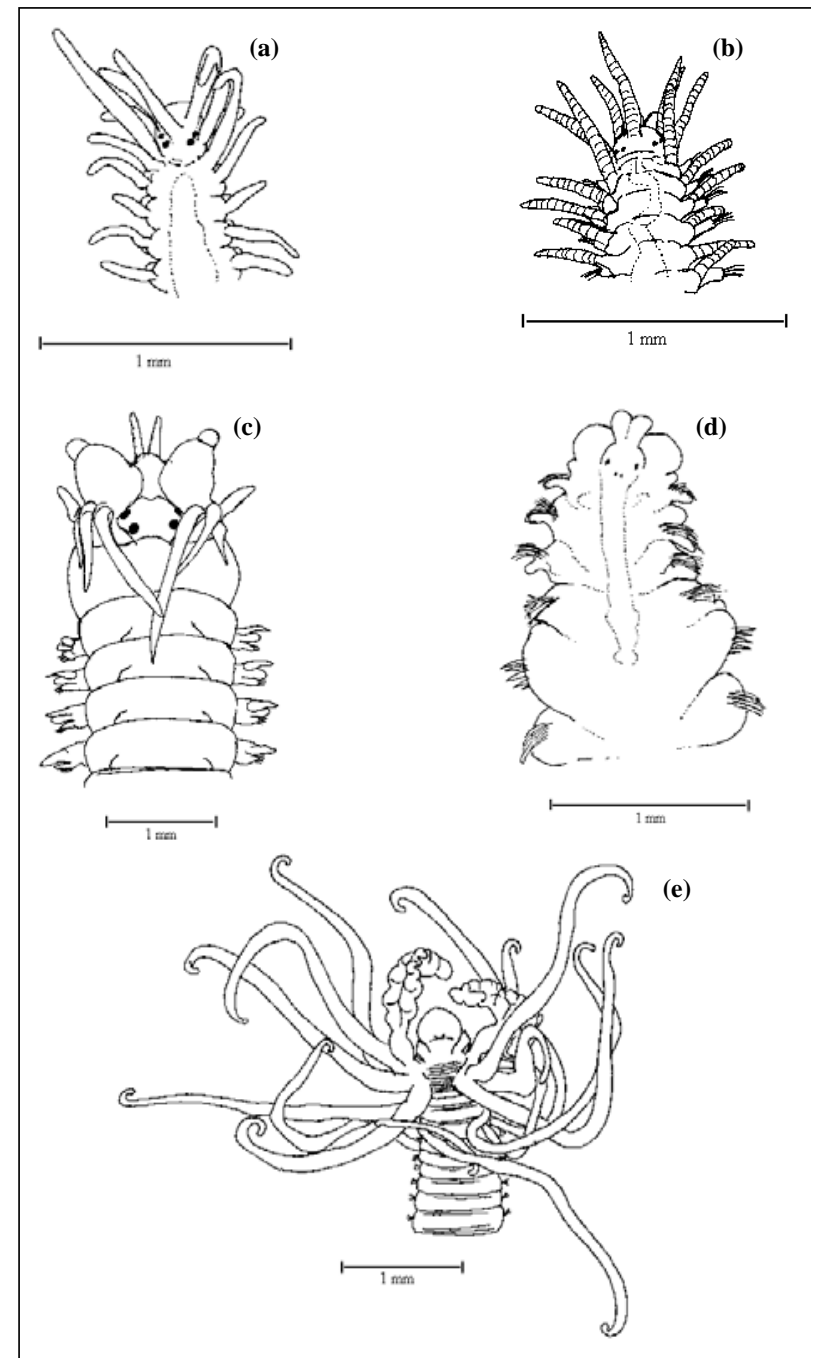

Figura 1

Extremo anterior en vista dorsal de Autolytus sp. (a), Typosyllis sp. (b), Nereis callaona (c), Polydora sp. (d) y Dodecaceria opulens (e). Dorsal view of anterior extreme of Autolytus sp. (a), Typosyllis sp. (b), Nereis callaona (c), Polydora sp. (d), and Dodecaceria opulens (e).

Tabla 2

Estadística descriptiva (Promedio, desviación estándar (DE), mínimo y máximo) de la biodiversidad Alfa de poliquetos asociados para los 30 cirripedios analizados. Se indica la abundancia de poliquetos (A), la riqueza de especies (R), la diversidad $H$ ' y uniformidad J' de Shannon-Weaver, la diversidad D de Simpson, y la diversidad N1 de Hill. Además, se indican los mismos descriptores de biodiversidad para la muestra total.

Descriptive statistics (mean, standard deviation (DE), minimum, and maximum) of the alfa biodiversity of associated polychaetes for the 30 cirripeds analyzed. We indicate the abundance of polychaetes (A), species richness (R), $\mathrm{H}^{\prime}$ diversity and $\mathrm{J}^{\prime}$ uniformity of Shannon-Weaver, Simpson's D diversity, and the N1 diversity of Hill. We also indicate these same biodiversity descriptors for the total sample.

\begin{tabular}{lcccccc} 
& A & R & $\mathbf{H}^{\prime}$ & $\mathbf{J}^{\prime}$ & D & N1 \\
\cline { 2 - 7 } Promedio & 26.4 & 6.5 & 0.64 & 0.78 & 0.27 & 12.33 \\
DE & 18.9 & 2.3 & 0.12 & 0.12 & 0.12 & 5.87 \\
Mínimo & 1 & 1 & 0.43 & 0.55 & 0.10 & 1.44 \\
Máximo & 70 & 12 & 0.88 & 0.97 & 0.50 & 26.37 \\
& & & & & & \\
\hline Muestra total & 793 & 19 & 0.77 & 0.61 & 0.28 & 18.84 \\
\hline
\end{tabular}




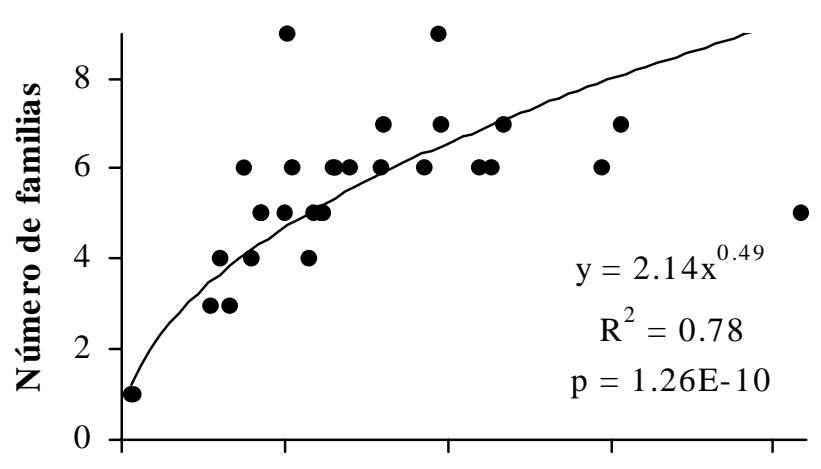

a
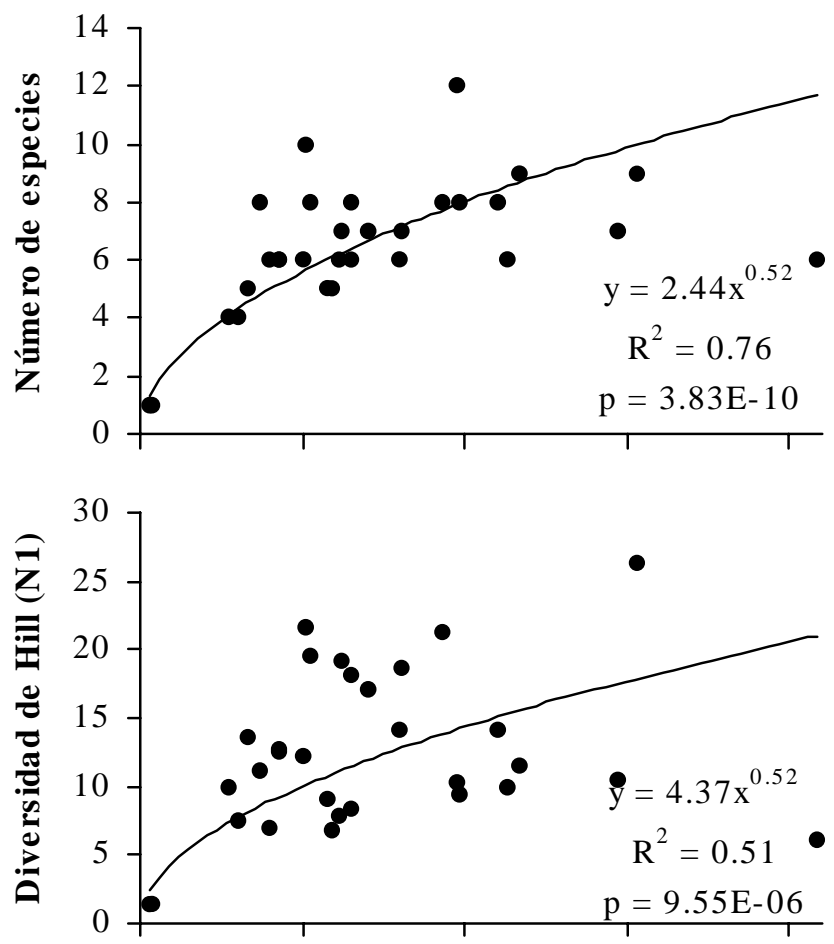

$\mathbf{b}$

C

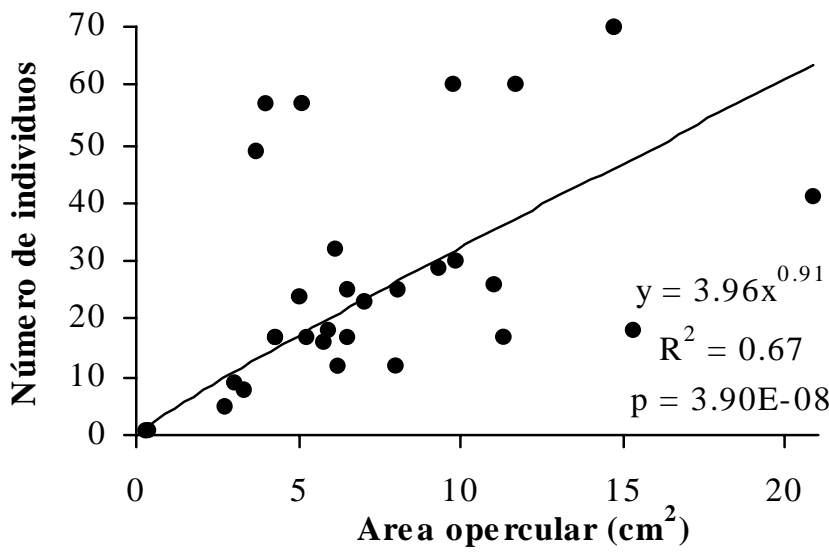

Figura 2

Curvas potenciales $\left(\mathrm{Y}=\mathrm{cX}^{\mathrm{z}}\right)$ de relación de la biodiversidad de poliquetos (a.- número de familias, b.- Número de especies, c.diversidad de Hill y d.- número de individuos totales) con respecto al tamaño de $A$. psittacus.

Potential curves $\left(\mathrm{Y}=\mathrm{cX} \mathrm{X}^{\mathrm{z}}\right)$ describing the relationship of polychaete biodiversity (a.-Number of families, b.-Number of species, c.-Hill diversity and d.-Number of total individuals) with respect to the size of $A$. psittacus. 


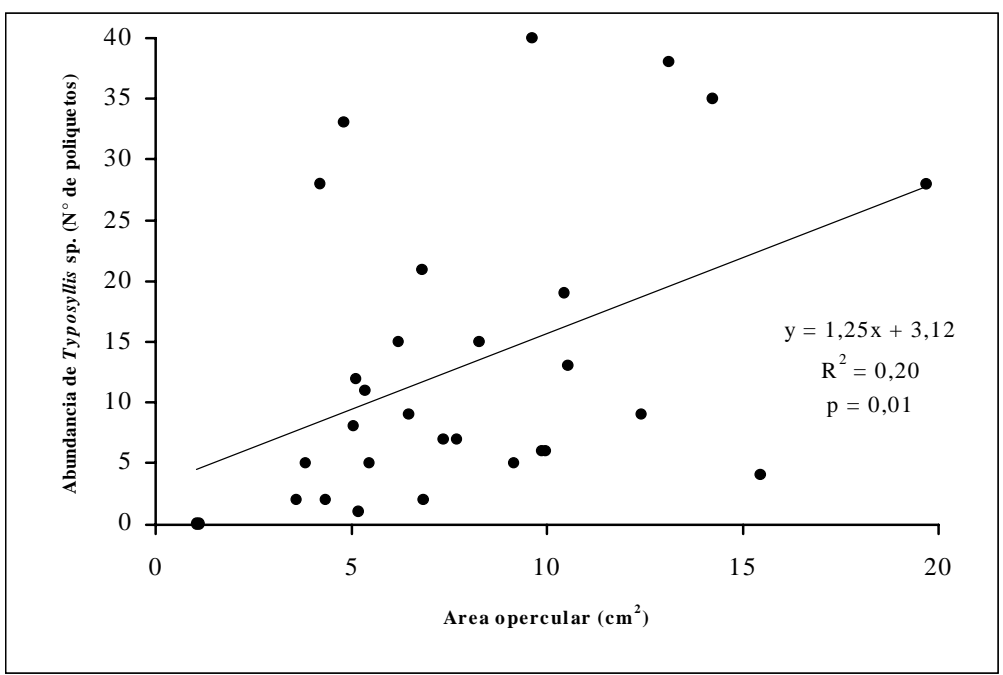

Figura 3

Relación entre la abundancia de Typosyllis sp. y el área opercular de $A$. psittacus.

Relationship between the abundance of Typosyllis sp. and the opercular area of A. psittacus.

1985 y 1986), en Crassostrea gigas (Tsuchiya \& Hirano 1985) y en un coral hermatípico (Tsuchiya et al. 1986), siendo los Nereididae los más abundante en bivalvos. Esta situación se repite en los poliquetos asociados a parches de Perumytilus purpuratus (Lamarck, 1819) y Semimytilus algosus (Gould, 1850) en Perú (Paredes \& Tarazona 1980), en España sobre hojas y rizomas del alga Posidonia oceanica (Alós 1983) y en Turquía con la esponja Sarcotragus muscarum Schmidt, 1864 (Cinar \& Ergen 1998). En el caso de los mitílidos de Perú la mayor abundancia estuvo dada por el nereidido Nereis callaona que también esta presente en el cirripedio A. psittacus y en parches de Pyura chilensis Molina, 1782 en Bahía Corral (Chile) (Zamorano \& Moreno 1975).

En el presente trabajo representantes de Syllidae mostraron las mayores

\section{Discusión y Conclusiones}

Entre los poliquetos que pueden establecer relaciones simbióticas del tipo comensal (Wargo \& Ford 1993) con los cirripedios se encontraron espionidos y cirratúlidos (perforadores), que pueden habitar sustratos calcáreos de organismos vivos y que en el presente estudio mostraron ser unos de los taxa más abundantes (Tabla 1). El principal representante de Spionidae en el presente trabajo pertenece al género Polydora que incluye a reconocidos perforadores de placas calcáreas de ostras (Loosanoff \& Engle 1943) y ostiones (Basilio et al. 1995), por lo que las placas calcáreas de $A$. psittacus constituirían un nuevo sustrato biológico en el cual habitan. También se ha señalado a Polynoidae como un taxa que forma asociaciones simbióticas tanto en Chile (Rozbaczylo \& Cañete 1993), como en otras partes del mundo (ej. Pettibone 1993, Britayev \& Zamishliak 1996, Jollivet et al. 2000), sin embargo en el presente estudio esta familia estuvo escasamente representada.

Al igual que lo observado en el cirripedio $A$. psittacus, Syllidae y Nereididae siempre han sido los taxa más abundantes asociados a sustratos biológicos, tales como tunicados, bivalvos, algas, corales, esponjas y agregaciones de tubos de otros poliquetos (Zamorano \& Moreno 1975, Paredes \& Tarazona 1980, Alós 1983, Tsuchiya \& Hirano 1985, Tsuchiya \& Nishihira 1985 y 1986, Tsuchiya et al. 1986, López \& Stotz 1997, Cinar \& Ergen 1998, Zamorano $2000^{1}$ ). Se ha informado sobre representantes de estas familias en Japón sobre parches de Mytilus edulis (L.) (Tsuchiya \& Nishihira abundancias, al igual que en los corales del Japón (Tsuchiya et al. 1986), en el alga Corallina officinalis L. (López \& Stotz 1997), en Pyura chilensis de Bahía Corral (Zamorano \& Moreno 1975) y en agregaciones de tubos de Pharagmatopoma moerchi en el intermareal rocoso de Mehuín (Zamorano 2000¹), específicamente con el género Typosyllis que también ha estado presente en Perumytilus purpuratus y Semimytilus algosus de Perú (Paredes \& Tarazona 1980).

El hecho que representantes de Syllidae y Nereididae presenten altas prevalencias y abundancias, es concordante con el hecho de que han sido descritos como numerosos y habitantes de sustratos duros y calcáreos (Barnes, 1987), al igual que Spionidae. Además son generalmente carnívoros y omnívoros con faringe eversible (Fauchald \& Jumars 1979), lo cual favorece su presencia en grietas desde las cuales se pueden alimentar (Tabla 1). Por otra parte, López \& Stotz (1997) los caracterizan como habitantes permanentes de determinados microhábitat, donde cumplirían todo su ciclo de vida. Esto último determinaría que habitualmente sean los grupo de mayor abundancia y diversidad en una gran variedad de sustratos, como en rocas y mezcla de arena-algas en la Isla de Pascua (Kohon \& Lloyd 1973).

El sustrato biológico fijo que constituye A. psittacus proporcionaría refugios al presentar grietas y orificios, por lo que sería un hábitat de fácil acceso para la colonización por poliquetos. De esta forma, A. psittacus no solo tiene importancia para el hombre como recurso económico (Bustamante \& Castilla 1987), sino también 
para los poliquetos que habitan en él, cuya biodiversidad podría ser perjudicada por la sobreexplotación comercial de $A$. psittacus. Cabe destacar, que en los últimos 10 años se extrajo entre 32 y 1160 toneladas sólo en la VIII región (SERNAP 19901999). Por lo que la descripción y cuantificación de la biodiversidad de poliquetos asociados a este hábitat (Tabla 2) permitirá hacer comparaciones con otros trabajo que se realicen en el futuro, además de servir como línea base para programas de vigilancia ambiental (Cañete et al. 2000), ya que las muestras de este estudio se obtuvieron en un sitio no contaminado de una de las bahías más contaminadas de Chile (Ahumada et al. 1989).

En la infracomunidad de poliquetos asociados a $A$. psittacus se destaca la presencia de Paleanotus chrysolepis, especie que en Chile sólo había sido registrada formalmente para Antofagasta (Rozbaczylo, 1985), y Dodecaceria opulens antes sólo registrada en el molusco murícido Concholepas concholepas (Bruguière, 1789) (Gibson, 1978) y en el pectínido Argopecten purpuratus Lamarck, 1819 (Sánchez \& Oliva $1997^{2}$, Cañete com. pers.). Aunque el género Dodecaceria también se ha registrado en Pyura chilensis (Zamorano \& Moreno 1975) y en grampones de Lessonia sp. (Villouta \& Santelices 1984).

La relación entre el tamaño de $A$. psittacus y la riqueza de poliquetos (Fig. 2) muestra que los cirripedios se comportarían como islas en las cuales aumenta la diversidad a medida que aumenta su tamaño, tal como lo plantea Kuris et al. (1980) desde el punto de vista de la biogeografía de islas en la relación parásitohospedero. De hecho, tanto animales y plantas que son parasitados podrían considerarse como islas y analizarse bajo las premisas del modelo de equilibrio de MacArthur \& Wilson (1963) (Jaksic 2001). Aunque en estos casos, al igual que en $A$. psittacus, la ecuación que empíricamente describe la conocida relación especiesárea presenta exponentes $\mathbf{z}$ (pendientes) mayores que en islas verdaderas (Lawton 1982).

Este aumento y tendencia a la saturación de especies descrita en $A$. psittacus, se relacionaría con el hecho de que los cirripedios más grandes tienen más tiempo de vida y en consecuencia un mayor tiempo de exposición a la colonización de nuevos poliquetos, además de un potencial mayor número de microhábitat (mayor número de grietas y orificios en la concha). Una relación semejante de aumento de la diversidad de la fauna asociada se observa con el aumento del tamaño en la colonia del coral hermatípico Pavona frondifera

\footnotetext{
2 Sánchez M. \& M. Oliva. 1997. Endoparásitos metazoos y comensales perforadores de Argopecten purpuratus Lamarck, 1819, en el norte de Chile: ¿Indicadores de unidades poblacionales?. XVII Congreso de Ciencias del Mar, Santiago, Chile, p. 147, Resumen.
}

(Tsuchiya et al. 1986), en parches de Mytilus edulis (Tsuchiya \& Nishihira 1985 y 1986), en parches de Crassostrea gigas (Tsuchiya \& Hirano 1985), en discos adhesivos del alga Lessonia nigrescens Bory (Vásquez \& Santelices 1984) y en la esponja Sarcotragus muscarum (Cinar \& Ergen 1998). Lo anterior se vería respaldado a escala poblacional por la relación positiva entre el tamaño del hospedador y la abundancia de Typosillys sp. (Fig. 3), situación que también fue registrada en Typosillis adamanteus korilensis Chlebovitsh en las costas de Japón por Tsuchiya \& Nishihira (1985), los cuales ven aumentar la abundancia de esta especie con el aumento del tamaño del parche de Mytilus edulis.

Finalmente, los poliquetos asociados a A. psittacus muestran patrones semejantes a los obtenidos en otras asociaciones reportadas en el litoral. La presencia de 19 taxa y una gran abundancia de algunos de estos (Tabla 1) marcan a los cirripedios como una importante fuente de hábitat y microhábitat para la biodiversidad de poliquetos, dentro de las comunidades de macroinvertebrados marinos donde estos habitan.

\section{Agradecimientos}

Los autores agradecen a Daniela Iriarte, Fabián Avilés, Hugo Díaz, Felipe Torres, José Gallardo, Luis Cruz (“El Beto”) y Paula Neill, por el apoyo dado en la realización del estudio. C.E. Hernández agradece el financiamiento recibido a través del Proyecto FIP (9649) y la beca de doctorado CONICYT. Finalmente, por su colaboración y sugerencias, a Yeny Rojas C. (Q.E.P.D.) y al Prof. Juan Cañete.

\section{Literatura Citada}

Ahumada R, A Rudolph, S Madariaga \& F Carrasco. 1989. Descripción de las condiciones oceanográficas de la bahía San Vicente y antecedentes sobre los efectos de la contaminación. Biología Pesquera 18: 37-52.

Alós C, A Campoy \& F Pereira. 1982. Contribución al estudio de los anélidos poliquetos endosimbiontes de esponjas. Actas II Simposio Ibérico de Estudios del Bentos Marino 3: 139-157.

Alós C. 1983. Anélidos poliquetos del Cabo de Creus. IIHojas de Posidonia oceanica. Publicaciones del Departamento de Zoología de Barcelona 9: 23-30.

Alós C. 1990. Anélidos poliquetos del Cabo de Creus (NE de España) facies de Corallina elongata Ellis \& Solander y de Cystoseira mediterranea (Feldmann). Miscelánea Zoológica, Barcelona 14: 17-28.

Barnes R. 1987. Zoología de los Invertebrados, 957 p. Interamericana, McGraw-Hill, México.

Barnes RSK \& RN Hughes. 1990. An introduction to marine ecology, 351 p. Blackwell Scientific Publications, Oxford. 
Basilio CD, JI Cañete \& N Rozbaczylo. 1995. Polydora sp. (Spionidae), un poliqueto perforador de las valvas del ostión Argopecten purpuratus (Bivalvia: Pectinidae) en Bahía Tongoy, Chile. Revista de Biología Marina (Valparaíso) 30 (1): 71-77.

Brown JH \& MV Lomolino. 1998. Biogeography. Second edition, 691 p. Sinauer Associates, Inc. Publishers, Sunderland, Massachusetts.

Britayev TA \& EA Zamishliak. 1996. Association of the commensal scaleworm Gastrolepidia clavigera (Polychaeta: Polynoidae) with holothurians near the coast of South Vietnam. Ophelia 45: 175-190.

Bustamante RH \& JC Castilla. 1987. The shellfishery in Chile: An analysis of 26 years of landings (1960-1985). Biología Pesquera 16: 79-97.

Cañete JI, GL Leighton \& FF Aguilera. 1999. Polychaetes from Aysén Fjord, Chile: distribution, abundance and biogeographical comparison with the shallow soft-bottom polychaete fauna from Antarctica and the Magellan Province. Scientia Marina 63: 243-252.

Cañete JI, GL Leighton \& EH Soto. 2000. Proposición de un índice de vigilancia ambiental basado en la variabilidad temporal de la abundancia de dos especies de poliquetos bentónicos de bahía Quintero, Chile. Revista de Biología Marina y Oceanografía 35: 185-194.

Carrasco FD \& VA Gallardo. 1989. La contaminación marina y el valor de la macroinfauna bentónica en su evaluación y vigilancia: Casos de estudio en el litoral de Concepción, Chile. Biología Pesquera 18: 15-27.

Cinar ME \& Z Ergen. 1998. Polychaetes associated with the sponge Sarcotragus muscarum Schmidt, 1864 from the Turkish Aegean coast. Ophelia 48: 167-183.

Darwin Ch. 1854. A monograph on the sub-class Cirripedia, 684 p. Printed for The Ray Society, London.

Fauchald K. 1977. The Polychaete Worms. Definitions and Keys to the Orders, Families and Genera. Natural History Museum of Los Angeles County, Science Series 28: 1190.

Fauchald K \& PA Jumars. 1979. The diet of worms: A study of polychaete feeding guilds. Oceanographic Marine Biology Annual Reviews 17: 193-284.

Feller RJ, SE Stancyk, BC Coull \& DG Edwards. 1992. Recruitment of polychaetes and bivalves: long-term assessment of predictability in a soft-bottom habitat. Marine Ecology Progress Series 87: 227-238.

Gibson PH. 1978. Systematics of Dodecaceria (Annelida: Polychaeta) and its relation to the reproduction of its species. Zoological Journal of Linnean Society 63: 275287.

Gotelli NJ. 1995. A Primer of Ecology, 206 p., Sinauer Associates, Inc. Sunderland, Massachusetts.

Hernández C. 1999. Fecundidad y ciclo de madurez sexual de Austromegabalanus psittacus (Molina, 1782) en la VIII región, 81 p. Tesis de Licenciatura en Biología Marina. Facultad de Ciencias, Universidad Católica de la Santísima Concepción, Concepción-Chile.

Hill MO. 1973. Diversity and evenness: A unifying notation and it's consequences. Ecology 54(2): 427-432.
Jaksic F. 2001. Ecología de Comunidades, 233 p. Ediciones Universidad Católica de Chile, Santiago, Chile.

Jollivet D, A Empis, MC Baker, S Hourdez, T Comtet, C Jouin-Toulmond, D Desbruyères \& PA Tyler. 2000. Reproductive biology, sexual dimorphism, and population structure of deep sea hydrothermal vent scale-worm, Branchipolynoe seepensis (Polychaeta: Polynoidae). Journal of the Marine Biologycal Association of the U.K. 80:55-68.

Kohn AJ \& MC Lloyd. 1973. Marine polychaete annelids of Easter Island. Int. Revue ges. Hydrobiol. 58(5): 691-712.

Kuris AM, AR Blaustein \& JJ Alió. 1980. Hosts as islands. The American Naturalist 116: 570-586.

Lawton JH. 1982. Vacant niches and unsaturated communities: a comparison of bracken herbivores at sites on two continents. Journal of Animal Ecology 51: 573595.

Little C \& JA Kitching. 1996. The biology of rocky shores, 240 p., Oxford University Press, Oxford.

Loosanoff VL \& JB Engle. 1943. Polydora in oysters suspended in the water. Biological Bulletin 85: 69-78.

López DA. 1994. Efecto de la estructura corporal de cirripedios en el resultado de interacciones bióticas, 181 p. Tesis Doctoral, Pontificia Universidad Católica de Chile, Santiago.

López CA \& WB Stotz. 1997. Descripción de la fauna asociada a Corallina officinalis L. En el intermareal rocoso de la costa de "Palo Colorado" (Los Vilos, IVRegión, Chile). Revista de Biología Marina y Oceanografía 32 (1): 17-35.

MacArthur RH \& EO Wilson. 1963. An equilibrium theory of insular zoogeography. Evolution 17: 373-387.

McAleece N. 1997. BioDiversity Professional V2. The Natural History Museum and The Scottish Association for Marine Science. http://www.nrmc.demon.co.uk/bdpro/ email: biodiversity@nhm.ac.uk.

Ojeda FP \& B Santelices. 1984. Invertebrate communities in holdfast of Macrocystis pyrifera. Marine Ecology Progress Series 16: 65-73.

Ojeda FP \& JH Dearborn. 1989. Community structure of macroinvertebrates inhabiting the rocky subtidal zone in the gulf of Maine: seasonal and bathymetric distribution. Marine Ecology Progress Series 57: 147-161.

Oyarzún C, FD Carrasco \& VA Gallardo. 1987. Some characteristics of macrobenthic fauna from the organic enriched sediments at Talcahuano, Chile. Cahiers de Biologie Marine 28: 429-446.

Paredes C \& J Tarazona. 1980. Las comunidades de mitílidos del mediolitoral rocoso del departamento de Lima. Revista Peruana de Biología 2 (1): 59-71.

Pettibone MH. 1993. Scaled polychaetes (Polynoidae) associated with ophiuroids and other invertebrates and review of species referred to Malmgrenia McIntosh and replaced by Malmgreniella Hartman, with descriptions of new taxa. Smithsonian Contributions to Zoology 538: 192. 
Rozbaczylo N. 1985. Los anélidos poliquetos de Chile. Indice sinonímico y distribución geográfica de especies. Monografías Biológicas 3: 1-284.

Rozbaczylo N \& JI Cañete. 1993. A new species of scaleworm, Harmothoe commensalis (Polychaeta: Polynoidae), from mantle cavities of two Chilean clams. Proceedings of the Biological Society of Washington 106 (4): 666-672.

SERNAP. 1990-99. Anuario estadístico de pesca. Servicio Nacional de Pesca. Ministerio de Economía, Fomento y Reconstrucción de Chile.

Shannon CE \& W Weaver. 1949. The mathematical theory of communication, 125 p., University of Illinois Press, Urbana.

Simpson EH. 1949. Measurement of diversity. Nature 163: 688.

Tsuchiya M \& YJ Hirano. 1985. Distribution of intertidal animal and associated fauna of the patch of the oyster Crassostrea gigas on the rocky shore of Mukaishima Islan, Hiroshima, southwestern Japan. Bulletin of the Biological Society of Hiroshima University 51: 3-9.

Tsuchiya M \& M Nishihira. 1985. Island of Mytilus as a habitat for small intertidal animal: effect of island size on community structure. Marine Ecology Progress Series 25: 71-81.
Tsuchiya M \& M Nishihira. 1986. Island of Mytilus edulis as a habitat for small intertidal animal: effect of Mytilus edulis age structure on the species composition of the associated fauna and community organization. Marine Ecology Progress Series 31: 171-178.

Tsuchiya M, Y Nakasone \& M Nishihira. 1986. Community structure of coral associated invertebrates of the hermatypic coral, Pavona frondifera, in gulf of Thailand. Galaxea 5: 129-140.

Vásquez JA \& B Santelices. 1984. Comunidades de macroinvertebrados en discos adhesivos de Lessonia nigrescens Bory (Phaeophyta) en Chile central. Revista Chilena de Historia Natural 57: 131-154.

Villouta E \& B Santelices. 1984. Estructura de la comunidad submareal de Lessonia (Phaeophyta, Laminariales) en Chile norte y central. Revista Chilena de Historia Natural 57: 111-122.

Wargo RN \& SE Ford. 1993. The effect odd shell infestation by Polydora sp. and infection Haplosporidium nelsoni (MSX) on the tissue condition of oysters, Cassostrea virginica. Estuaries 16(2): 229-234.

Zamorano JH \& CA Moreno. 1975. Comunidades bentónicas del sublitoral rocoso de bahía Corral: I. Area mínima de muestreo y descripción cuantitativa de la asociación de Pyura chilensis Molina. Medio Ambiente 1(1): 58-65.

Zar JH. 1996. Biostatistical Analysis, 867 p. $3^{\text {nd }}$ ed, Prentice Hall, Englewood Cliffs. New Jersey. 